\title{
Acute primary angle closure-treatment strategies, evidences and economical considerations
}

\author{
Poemen P. Chan ${ }^{1,2} \cdot$ Jason C. Pang ${ }^{1} \cdot$ Clement C. Tham ${ }^{1,2,3}$
}

Received: 9 August 2018 / Revised: 23 September 2018 / Accepted: 30 September 2018 / Published online: 22 November 2018

(c) The Royal College of Ophthalmologists 2018

\begin{abstract}
Acute primary angle closure requires emergency management that involves a rapid lowering of the intraocular pressure and resolution of relative pupil block - the most common mechanism of angle closure. Emergency strategies for lowering intraocular pressure include medical treatment and argon laser peripheral iridoplasty. Anterior chamber paracentesis and diode laser transcleral cyclophotocoagulation may be considered in special situations. Relative pupil block can be relieved by peripheral laser iridotomy and primary lens extraction; the latter is a more effective treatment according to the results of clinical trials. However, primary lens extraction can be technically demanding in the acute setting. Peripheral laser iridotomy has a role in relieving pupil block and should also be considered in most cases. Lens extraction may be combined with procedures such as goniosynechialysis, trabeculectomy or endoscopic cyclophotocoagulation. In this review, we aim to discuss the available evidence regarding the different treatment modalities. We also discuss the economic consideration, including cost-effectiveness and life expectancy, in the management of acute primary angle closure.
\end{abstract}

\section{Introduction}

Acute primary angle closure (APAC) is usually caused by an abrupt closure of the trabecular meshwork in the anterior chamber angle that leads to a sudden rise in intraocular pressure (IOP). It is understood that an exaggerated pupillary block disturbs the natural aqueous flow from the posterior to the anterior chamber, creating a pressure gradient and leads to a forward bowing of the peripheral iris. The iris apposes to the trabecular meshwork, and thus causes an acute angle closure. APAC is a subgroup of angle closure disease characterised by a sudden onset of headache, blurred vision, seeing halos around lights, corneal oedema, middilated pupil, eye pain and redness. The treatment outcomes

$\triangle$ Clement C. Tham

clemtham@cuhk.edu.hk

1 Department of Ophthalmology \& Visual Sciences, The Chinese University of Hong Kong, Hong Kong SAR, People's Republic of China

2 Hong Kong Eye Hospital, Kowloon, Hong Kong SAR, People's Republic of China

3 Prince of Wales Hospital, Shatin, N.T., Hong Kong SAR, People's Republic of China of APAC are quite different between Asian (more pigmented iris) and Caucasian eyes (usually less pigmented iris). Laser peripheral iridotomy (LPI) tend to be less effective in controlling the IOP in Asian eyes with APAC. Asian also has a much higher incident rate of APAC compare to the Caucasian population - with the crude incidence rate of 12.2 and 10.4 per 100,000 people per year in the above 30-year-old population of Singapore and Hong Kong, respectively [1, 2]. This is higher than the average incidence rate of 3.9-4.1 cases per 100,000 people per year in the European regions [3-5].

In APAC, both LPI and primary lens extraction by phacoemulsification and intraocular lens implant (phaco/ IOL) were demonstrated to be effective to control IOP elevation. The latter has been shown to be the more effective treatment than LPI for IOP reduction at the early and mid-term IOP control. Together with the advancement of phaco/IOL technique, primary lens extraction is the more popular choice of treatment nowadays. However, operating on an eye with early aborted APAC is technically challenging and may increase the risk of complications because of the presence of corneal oedema, inflammation, shallow anterior chamber, floppy iris and unstable lens. Furthermore, "the best time window" for performing lens extraction after an APAC attack remains uncertain [6]. The longterm results (e.g. more than 5 years) of early lens extraction 
compared to the conventional LPI are also unknown. In this review, we summarise the approach of treating APAC at the initial acute stage and review the studies that consider IOP control in the mid and long term. We would also touch on the role of goniosynechialysis, trabeculectomy and endoscopic cyclophotocoagulation in treating APAC, as well as considering the economic aspect of treatment.

\section{Treatment approach and considerations}

The principle of treatment for APAC aims at (1) initial rapid reduction of IOP in order to limit optic nerve damage, followed by (2) elimination of pupil block, which reduces the risk of recurrent attack and the risk of progression to the chronic form of primary angle closure glaucoma (PACG).

\section{(1) Initial rapid reduction of IOP}

Rapid reduction of IOP is an important initial step of treating APAC because it prevents further glaucomatous optic nerve damaged. It also reduces pain and corneal oedema, which allows implementation of more definitive treatments; namely LPI and lens extraction. The use of topical and systemic IOP-lowering medication is usually sufficient to achieve the initial IOP control. Amongst the topical IOP-lowering medications, pilocarpine has a physiological advantage to counteract the angle closure mechanism by inducing miosis, pulling the peripheral iris away from the trabecular meshwork in order to break a mild acute attack. Notice that pilocarpine of high concentration (e.g. pilocarpine 4\%) may also lead to increase vascular permeability, which could increase vascular congestion of the iris, pushes the lens-iris diaphragm more anteriorly and aggregates the pupillary block. APAC could also lead to pupillary sphincter ischaemia, rendering it irresponsive to the effect of pilocarpine. Multiple medications might be required to abort the APAC episode. However, a lot of these patients are elderly patients and some might have multiple medical conditions. They might not tolerate the potential side effects of these drugs, especially in the situation where systemic medications are required. Carbonic anhydrase inhibitor and hyperosmotic agents could lead to paraesthesia, drowsiness, confusion, loss of appetite, polydipsia and polyuria. For susceptible patients (e.g. patients with renal failure), it could lead to serious complications such as metabolic disturbance, respiratory failure, pulmonary oedema, congestive heart failure, acute renal failure and even intracranial haemorrhage. Rare but severe systemic complications include Stevens-Johnson syndrome, blood dyscrasias and anaphylactic reactions. A significant proportion of APAC patients do not respond to medical treatment alone adequately. In these cases, other interventions might be considered to achieve rapid IOP reduction.

Argon laser peripheral iridoplasty (ALPI) can mechanically open up the angle in APAC [7, 8]. A series of studies have proven its effectiveness and safety when applying to eye with APAC [9-12]. A randomised control trial (RCT) has also demonstrated that ALPI is significantly more effective in reducing IOP in APAC at $15 \mathrm{~min}, 30 \mathrm{~min}$ and $1 \mathrm{~h}$ after the start of treatment when compared to conventional systemic medication [13]. Although this difference in IOP became statistically insignificant from 2-h onwards, ALPI arguably has more advantages at the early stage treatment because of its superior efficacy in lowering IOP and more favourable side effect profile. However, the follow-up study of the same group of patients, that compared the clinical outcome of ALPI against systemic medication in the mid-term (mean follow-up duration of 15 months), suggested that there were no statistically significant differences in IOP control between the two groups [14]. Nonetheless, ALPI is an opinion to consider in the acute stage of the disease. In clinical practice, we should also be aware of the technique and potential complication of applying ALPI. The eye should be pre-treated with topical anaesthetic and pilocarpine. One should aim at producing a contraction burn at the most peripheral portion of the iris as possible with the setting of large spot size, long duration and low power Argon laser (e.g. $500 \mu \mathrm{m}$ spot size, $0.5 \mathrm{~s}$ duration, start from a power of $240 \mathrm{~mW}$ ) through an Abraham iridotomy contact lens. One of the potential complications of ALPI is corneal endothelial burn because of the proximity of peripheral iris and the cornea. The risk of complication is higher especially when APAC are usually handled by junior ophthalmologists as emergency cases, who might have limited experience in performing ALPI for eyes with shallow anterior chamber and corneal oedema. ALPI should be contraindicated in case of severe corneal oedema, flat anterior chamber and synechial angle closure.

Immediate anterior chamber paracentesis has also been proposed as an alternative procedure during the initial stage of APAC [15]. It can be a helpful procedure if maximally tolerated medication cannot adequately reduce IOP, and when ALPI or LPI is not possible (e.g. unavailability of laser, severe corneal oedema that blurred the anterior chamber view for laser application). It allows rapid reduction of IOP and corneal oedema, which makes early intervention by laser therapy possible. Anterior chamber paracentesis may pose many technical difficulties [16], mainly the concern of iris and lens damage from the paracentesis slit knife and potential further closure of the angle upon decompression. Another safe and easy alternative to perform anterior chamber paracentesis is the use of a half-inch 30-gauged needle [17]. During the 
procedure, topical anaesthetic eye drops is applied and the conjunctival sac and lashes are prepared with povidone iodide solution. After rinsing out the povidone, the patient is positioned at the slit lamp and the lids are held open with a speculum. The 30-gauged needle hub, which had been bent into half an inch, is grasped between the thumb and forefinger and the back of the hand rested on the patient's cheek for stabilisation. The tip of the needle then enters the cornea inferotemporally, thought an adequately long intrastromal cornea track and enters into the anterior chamber. Notice that the needle is orientated such that it is parallel to the iris plane and directed away from the lens. This way it avoids accidental puncturing of the intraocular structures. The needle is withdrawn after $10 \mathrm{~s}$ of the anterior chamber entre. Throughout the procedure, the needle is used without a syringe. The pressure necessary to force fluid through a half-inch 30-gauged needle is about $12 \mathrm{mmHg}$ so that the decompression stops automatically at that pressure and the anterior chamber would not flatten. The needle track is also self-sealed [17]. This technique could be considered if there is adequate space in the anterior chamber. It is important to note that anterior chamber paracentesis alone only temporarily reduce the IOP; the APAC episode may recur if the pupil block mechanism is not resolved promptly.

In cases were APAC could not be controlled by the methods mentioned above, alternative strategies could also be used. Diode laser transscleral cyclophotocoagulation (DLTSC) was reported to be a possible alternative in treating APAC [18, 19]. This allows more rapid lowering of IOP and reduces corneal oedema for further intervention. DLTSC achieves IOP-lowering effect by direct thermal destruction of the ciliary epithelial cells that produce aqueous. It may also induce localised inflammation that leads to a temporarily ciliary body shut down and/or increase uveoscleral outflow. However, it could lead to pain, inflammation and visual loss. Micropulse transscleral cyclophotocoagulation (Iris Medical Instruments, Mountain View, CA, USA) is also another alternative with potentially better safety profile $[20,21]$. In the case of refractory APAC, especially for patients with multiple medical conditions that present with poor visual acuity, it is important to perform careful examination and investigation before further treatment. This includes fundi examination (if possible), B scan and ultrasound biomicroscopy. The aim is to look for secondary causes of acute angle closure such as lens subluxation [22] and haemorrhage of the posterior segment [23-27]. Massively detached choroid and retina due to haemorrhage could lead to forward displacement of the lens-iris diaphragm. In these situations, further invention (e.g. lens extraction) could lead to devastating complications, such as intraoperative suprachoroidal haemorrhage.

\section{(2) Elimination of pupil block}

Once the acute attack has been overcome, the aim of treatment is to prevent further acute and chronic angle closure by eliminating the pupil block mechanism. This can be achieved by primary lens extraction or creating a peripheral iridotomy(PI). Nevertheless, early phaco/IOL is generally demonstrated to be the more effective approach than LPI in terms of preventing progression to chronic PACG and reducing the use of medication [3, 32-36].

\section{Primary lens extraction}

Phaco/IOL deepens the anterior chamber and eliminates pupil block [28, 29]. Overall, patients with APAC had a $71 \%$ reduction from presenting IOP and rarely require long-term glaucoma medication when the surgery was performed soon after medical reduction of IOP [30]. Trabeculectomy after phaco/IOL was uncommon [30]. There are only several studies on this topic and that limits our assessment (Table 1). Jacobi et al. [29] provided some early evidence to justify early phaco/IOL. In this prospective, nonrandomised comparative trial, 43 German patients with APAC were treated by phaco/IOL and 32 patients were treated by conventional surgical peripheral iridectomy (SPI). The mean follow-up period was 10.2 months. APAC patients treated by primary phaco/IOL did significantly better in terms of IOP reduction and best-corrected visual acuity. They also required fewer antiglaucoma medications and less additional surgical interventions compared to the patients who underwent SPI.

A prospective RCT by Lam et al. [31] supported this finding. It compared the effects of primary phaco/IOL versus LPI in the prevention of IOP rise in patients soon after APACs were aborted. The mean time between abortion of attack and phaco/IOL and LPI were 5.7 days and 4.3 days, respectively. 31 patients were recruited in each arm. One patient from each group passed away before the end of the study. At 18 month, the early phaco/IOL group demonstrated a significantly lower prevalence of IOP elevation, required less glaucoma medications to maintain IOP at $<21 \mathrm{mmHg}$, larger degrees of open angle and less extensive peripheral anterior synechiae (PAS) on gonioscopy compared to the LPI group. However, there were no statistically significant differences in visual acuity, vertical cup to disc ratio (VCDR), median deviation (MD) and pattern standard deviation (PSD) on visual field (VF) between the two groups. None of these patients required further surgery to control IOP.

Later, Husain et al. [32] performed an RCT that compare the 2-year efficacy of primary phaco/IOL with LPI in the early management of APAC and coexisting cataract. Patients with APAC that had the IOP lowered to $\leq 30 \mathrm{mmHg}$ 


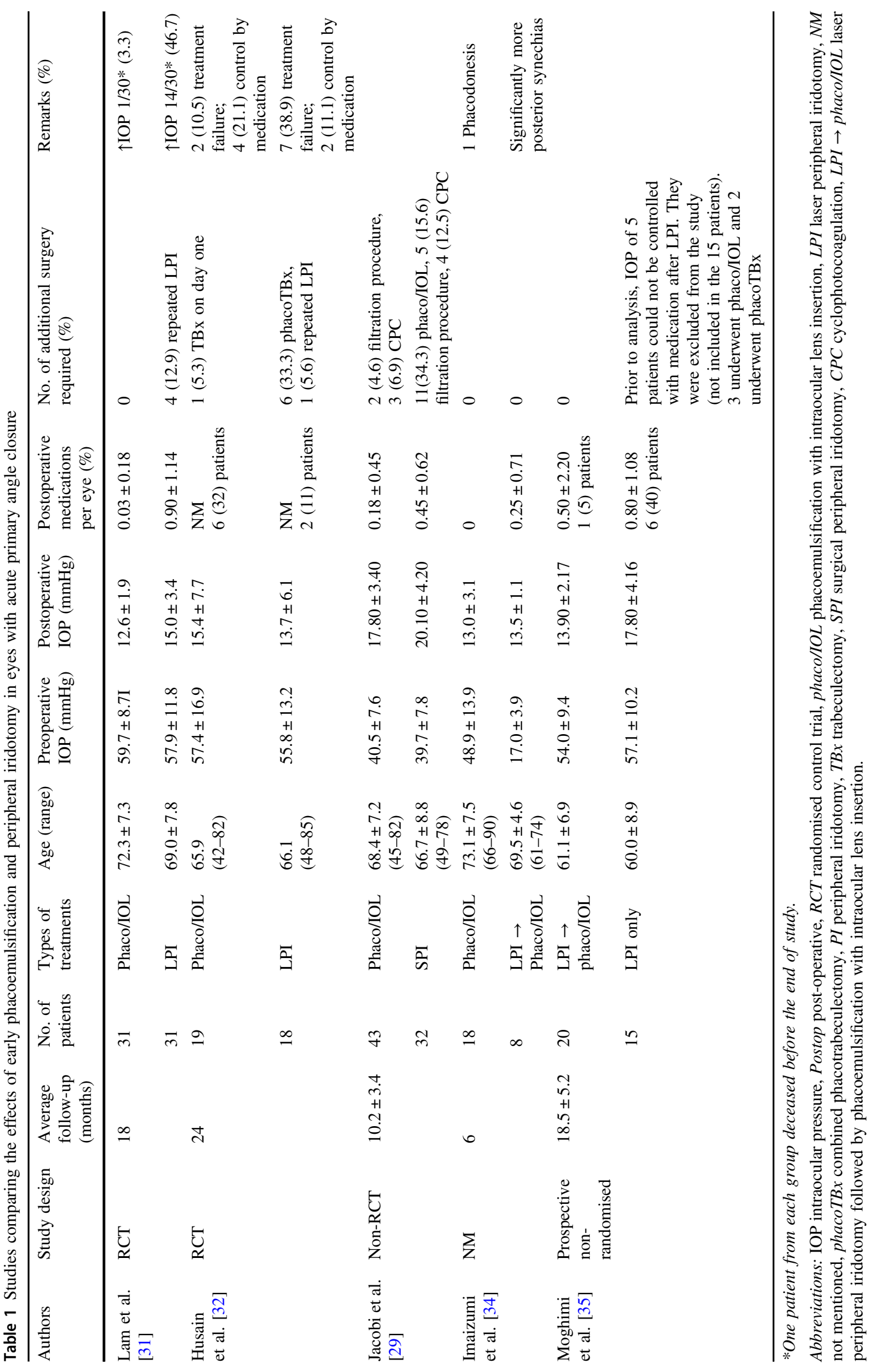


by medications within $24 \mathrm{~h}$ were treated either with LPI 72 $\mathrm{h}$ after the medical treatment or by phaco/IOL 5 to 7 days after the IOP was lowered. 18 patients and 19 patients were randomised to LPI and primary phaco/IOL group, respectively. Failure of IOP control was defined as IOP between 22 and $24 \mathrm{mmHg}$ on 2 occasions (readings were taken within 1 month of each other) or IOP $\geq 25 \mathrm{mmHg}$ on 1 occasion after week 2. Failure was also defined as loss of light perception attributable to glaucoma, the necessity for further operative intervention for glaucoma or recurrence of APAC. There was 1 patient who had IOP $>30 \mathrm{mmHg}$ on day 1 after operation in the phaco/IOL group. Whereas for the LPI group, there was 1 transient haemorrahge, 1 corneal burn and 3 cases required repeated LPI because of closure of the initial LPI. At 2-year, there were significantly less treatment failure in the phaco/IOL group $(2 / 19$ [10.5\%]) compared to the LPI group (7/18 [38.9\%]; $P=0.029)$. Six patients in the phaco/IOL group required IOP-lowering medications, of which 2 were considered failures because of high IOP. Whereas 7 out of 18 patients were classified as failures in the LPI group - 6 underwent combined phacotrabeculectomy with Mitomycin C and 1 underwent repeated LPI because of APAC recurrence (the initial LPI was noted to have closed). Another 6 patients in the LPI group underwent cataract surgery because of decreased visual acuity and were not for IOP control - they were not classified as treatment failure. The study had very strict recruitment criteria and was targeting APAC with coexisting cataract; subjects would only be recruited if they have a best-corrected visual acuity of $\leq 6 / 15$. That was one of the reasons why the authors were unable to recruit more patients then they initially intended to do. The authors concluded that phaco/IOL resulted in lower rate of IOP failure at 2 years compared with LPI if it is performed within the first week in patients with APAC and coexisting cataract.

Despite the demonstrated advantage of early phaco/IOL, lens extraction in eyes with APAC is known to be technically demanding. The patients are often anxious and the eyes are usually inflamed, with shallow anterior chamber depth, poor surgical view due to corneal oedema, high IOP, small pupils, floppy iris and sometime unstable lens. Under such condition, topical anaesthesia may not be sufficient for patients' comfort. This was reflected in Jacobi et al. [29] study, in which 5 out of 43 patients who underwent primary phaco/IOL had to undergo general anaesthesia because of anxiety. Intraoperative complication such as iris bleeding and corneal oedema could occur [31, 32]. The challenges of operating in this setting must be taken into account [33]. There is a higher risk of postoperative complication, such as cornea decompensating, wipe-out syndrome and posterior capsular rupture. The benefit of early lens extraction must be balanced with the risk of the operation.
Therefore, it is not an obligation to perform early primary phaco/IOL if the eye is not in good condition. LPI is still a very helpful technique to relief pupil block. A patent $\mathrm{PI}$ is effective in lowering IOP at the acute stage and preventing recurrence of APAC. It should be considered as the treatment of choice if primary lens extraction is difficult. A successful initial LPI probably would not jeopardise the effect of the subsequent lens extraction procedure. Imaizumi et al. [34] compared the results of phaco/IOL for 18 eyes that presented with APAC (i.e. no prior LPI) and 8 eyes that had been treated by LPI because of previous APAC (i.e. aborted APAC). At 6 month, there were no statistically significant differences in terms of IOP and medication use between the two groups (Table 1). This is similar to the result of a subgroup of patients in a nonrandomised comparative prospective study in Iran [35] (Table 1). In this study, 20 patients who had APAC and had been treated by LPI, underwent phaco/IOL due to the presence of significant cataract. After a mean follow-up period of 18.5 months, the mean postoperative IOP of the group was $13.9 \mathrm{mmHg}$ and the mean number of medication use was 0.5 bottle. These study demonstrated that prior LPI probably would not jeopardise the result of the subsequent phaco/IOL. In Table 1, we have also listed other studies that involved early phaco/IOL for APAC. These studies have follow-up periods of up to 24 months. The effect of the two treatment modalities (LPI and phaco/IOL) beyond 2 years is unknown. The "best time window" for performing lens extraction after an APAC attack is also unknown [6]. With these uncertainties in mind, surgeons should weight-over the risk and benefit before deciding to treat newly aborted APAC by phaco/IOL or LPI.

\section{Laser peripheral iridotomy}

Both SPI and laser peripheral iridotomy (LPI) are effective means of lowering IOP at the initial stage of the acute attack. For Caucasian eyes with APAC, IOP could be sufficiently controlled with SPI or LPI in up to $65-76 \%$ of the time; with the additional use of medical therapy, this rate could be up to $84-99 \%$ [36-40]. LPI is now preferred because it is relatively non-invasive, easy to perform on outpatient bases, and has a lower risk of complications [41]. Neodymium-doped yttrium aluminium garnet (Nd:YAG) laser alone is sufficient to create LPI for Caucasian but not sufficient for Asian eyes that have more pigmented iris. It is important to note that sequential argon laser and Nd:YAG laser should be performed for the latter; this allows effective PI formation whilst minimising tissue damage to the heavily pigmented iris [42-44].

When sequential argon and Nd:YAG laser is applied to Asian eyes, LPI could also be an effective treatment modality of controlling IOP at the initial stage of the APAC. 
This is demonstrated in a retrospective study done by Aung et al. (Table 2) [45]. In all, 111 eyes that presented with APAC and had undergone LPI were reviewed. Apart from 1 eye that required primary trabeculectomy because of non-patent LI, all the other eyes had IOP reduction to $<21 \mathrm{mmHg}$ without medication after the successful LPI. However, in the long-term, the results of LPI may not be as satisfactory. In the same study [45], which has a mean follow-up period of 50.3 months, only 46 out of the 110 eyes $(41.8 \%)$ had no subsequent increase in IOP on follow-up. A total of 64 eyes (58.2\% of 110) developed elevated IOP during the follow-up period - 49 eyes $(44.5 \%$ of 110) developed increase IOP within 6 months, 5 eyes (4.5\% of 110) in between 6 months to 1 year, and 10 eyes (9.2\% of 110) after 1 year of APAC presentation. 26 eyes (23.6\% of the 110 ) were controlled with topical medication, 36 eyes $(32.4 \%$ of the 110$)$ required trabeculectomy and the remaining two eyes had already lost light perception at presentation. The relatively poor outcome was echoed by a cross-sectional study of the same group [46] (Table 2). They demonstrated that $17.8 \%$ of the subjects were blind in the attacked eye and almost half had glaucomatous optic nerve damaged several years after APAC. The vision was also reduced in a large number of individuals, mainly because of coexisting cataract. Similarly, a prospective observational case series that aimed to evaluate the changes in the configuration of the drainage angle 1 year after APAC, showed that 19 out of 44 subjects (43\%) developed IOP elevation during the follow-up period and required additional glaucoma medication [47]. Even in Caucasian patients, up to $56 \%$ of the patients who had APAC required further IOP-lowering medication or procedures after LPI [48].

However, the outcomes of LPI were more satisfactory in other studies. Tan et al. [49] reviewed 42 eyes with APAC. 41 eyes achieved persistently patent PI and one eye required phacoemulsification after two unsuccessful attempts of LPI. The mean follow-up period was 27.3 months. Nine eyes (21.4\% of 42 eyes) developed an increase in IOP within a mean of 11.9 months after resolution of APAC. They were initially treated with topical medication with resultant IOP of $<21 \mathrm{mmHg}$. Out of these 9 eyes, 7 eyes ( $16.7 \%$ of 42 eyes) underwent combined phaco/IOL and trabeculectomy with Mitomycin-C and one eye underwent combined phaco/IOL and glaucoma drainage device insertion. At the final follow-up, the mean IOP was $13.3 \mathrm{mmHg}$ and none of the eyes (including those that underwent surgery) required additional topical medication. Similarly, in the Lai et al series [14] that included 79 APAC eyes (of 71 patients, they had either undergone ALPI or systemic medications prior to LPI), only 1 eye required cataract extraction within 6 months after the acute attack. This eye was operated because of chronic angle-closure glaucoma development. The mean follow-up duration was 15.7 months. There were no statistically significant differences in the mean IOPs and the requirements for glaucoma medication between the ALPI group and the medically treated group. The mean IOPs were $13.6 \mathrm{mmHg}$ and $14.7 \mathrm{mmHg}$, respectively, whilst the mean number of medication were 0.3 and 0.5 , respectively (Table 2).

The results of other studies mentioned in Table 1 also suggested that LPI could be an effective approach to control IOP in the mid-term. For instance, the patients who only underwent LPI in Lam et al. [31] and Moghimi S et al. [35] did not require additional surgery after a mean follow-up period of 18 months. In the Lam et al. series [31], the mean number of medications required to maintain IOP $\leq 21 \mathrm{mmHg}$ was significantly higher in the LPI group (0.90) compared to the primary phaco/IOL group (0.03) at 18-month. In the 10-year follow-up, 2 patients from the LPI group had severe visual acuity loss $(<0.1$ Snellen visual acuity) in the LPI group and none in the phaco/IOL group. Overall, there was no significant difference in terms of the visual field results between the two groups at the 10-year follow-up (unpublished data).

\section{Role of goniosynechialysis, trabeculectomy and endoscopic cyclophotocoagulation}

It has been shown that the use of viscoelastic during phacoemulsification could lead to a certain extent of PAS breakdown and achieve an associated IOP reduction [50]. Goniosynechialysis was described as an effective intervention when performed in combination with phaco/IOL in the case of angle closure [51-53]. This can also be combined with laser iridoplasty to prevent PAS reattachment $[54,55]$. However, the application of goniosynechialysis was not described specifically for APAC. It is also known that irreversible damage to the trabecular meshwork occurs especially in chronic synechial closure [56]. Therefore, we do not recommend aggressive goniosynechialysis for eyes with APAC because this could lead to surgical complications (e.g. hypaemia and fibrinoid anterior chamber reaction) without additional IOP reduction. Furthermore, eyes that had an acute on chronic type of presentation together with extensive glaucomatous optic neuropathy are probably more susceptible to damage. Aggressive goniosynechialysis could lead to IOP fluctuation during the procedure and thus lead to wipe-out syndrome.

Trabeculectomy alone may further shallow the anterior chamber and aggravate the angle closure. The success rate of trabeculectomy alone is as low as $56.2 \%$ percent in patients with medically unresponsive APAC [57]. Therefore, trabeculectomy alone is not recommended. After the acute phase of APAC, combined phacotrabeculectomy may 


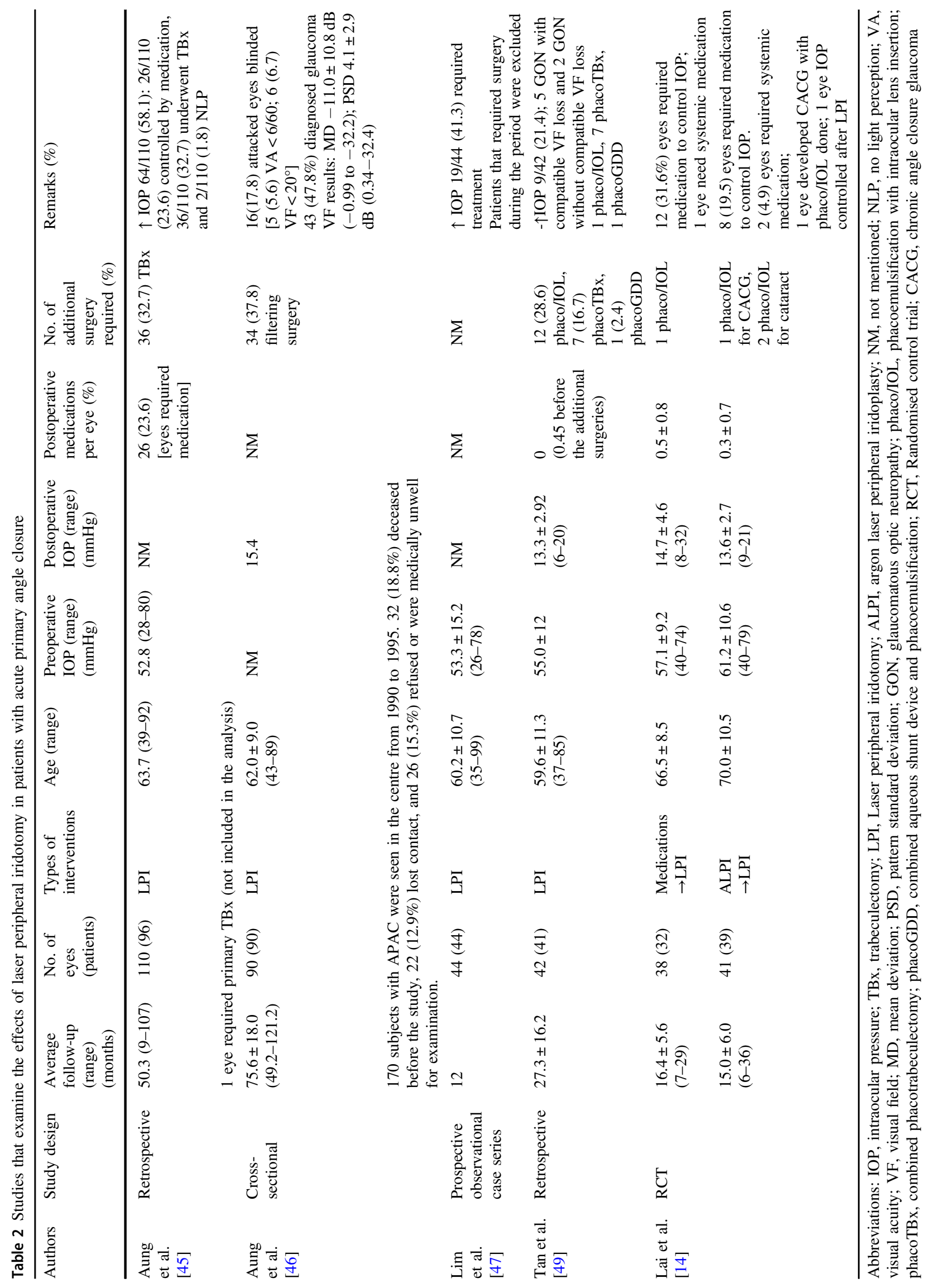


be considered, especially if the IOP is poorly controlled by medication [58, 59].

Endoscopic cyclophotocoagulation (ECP) has been recently introduced as a treatment modality for glaucoma. It provides a more direct and targeted method of performing cyclophotocoagulation of the ciliary body. It achieves IOP lowering by reducing aqueous production. It should be performed in combination with phaco/IOL. A study also demonstrated that angle in areas treated with ECP were open with a corresponding flattened ciliary process, suggesting that it could cause anatomical changes on top of the reduction of aqueous production [60], which could be helpful if there is a component of plateau iris configuration in the APAC eye, although its effectiveness in this situation requires further study to verify.

\section{Economical considerations in the management of APAC}

Cost-effectiveness of treatment is less mentioned in APAC. One study reported the direct cost of treating APAC [61]. The study was performed in the era before the popularity of prostaglandin analogue and before the publications of the RCTs mentioned in Table 1 [31, 32]. It suggested that treating APAC produced a substantial financial burden on society and individuals. The results showed that, over a 5-year horizon, the total cost of patients who required trabeculectomy because of poorly controlled IOP was similar to patients who had adequate control with chronic use of medication. The authors also predicted that medical treatment would be a more expansive opinion than surgery. Recently, our cost-effectiveness analysis of phacoemulsification versus combined phacotrabeculectomy for treating primary angle closure glaucoma demonstrated that the cost-effectiveness was sensitive to the cost fluctuation of medication but was insensitive to the cost of surgery [62]. We suggested that, in the long-term, the cost of medication is probably the most important factor to determine the total cost of treatment [62]. Therefore, in terms of cost-effectiveness, early phaco/IOL (with or without initial LPI) that aims at medication reduction is probably preferable. Of course, the technical difficulties of the surgery should be taken into consideration and should be operated on by experienced surgeon. The potential benefit of uneventful phaco/IOL in terms of refractive error correction and reduction of topical medication side effects may also improve patients' quality of life [63].

It is also important to consider life expectancy and patients' health status when making the treatment decision. Many patients who have APAC are elderly subjects with an average age of above 60 (Tables 1 and 2). In the cross- sectional case series of Aung et al. [46], 32 patients (19\% of 170) were deceased before the time of the examination (mean duration of evaluation from the time of APAC episode was 6.3 years) and numerous patients were medically unwell for examination. Life expectancy also influences the cost-effectiveness of a particular treatment [64-66]. Therefore, one should also take the overall health status of the patient into account when making the treatment decision.

\section{Conclusion}

Prompt reduction of IOP is important for APAC at presentation. This could mostly achieve by medical therapy. ALPI and anterior chamber paracentesis are also effective opinions. If the APAC is unresponsive to these treatments, careful evaluation is important and one should look for secondary causes of acute angle closure. Once the acute episode is aborted, primary phaco/IOL or LPI should be performed in order to eliminate pupil block. Overall, primary phaco/IOL is a more effective treatment modality in terms of long-term IOP control and reduction of medication use but it is technically more demanding. LPI is also an effective modality in the acute setting. However, the results might not be as satisfactory in the longer term. An initial LPI for eyes with APAC probably would not jeopardies the results of the subsequent phaco/IOL. The optimum timing for lens extraction remains unknown and further study is required.

Trabeculectomy alone is not a treatment opinion for APAC. Goniosynechialysis and ECP could be helpful in selective cases. These procedures should be combined with phaco/IOL. APAC produces a substantial financial burden. Reduction of cost of medication is a useful approach for reducing the total cost of treatment. Therefore, early phaco/ IOL may be a cost-effective approach but this requires verification by future studies. Life expectancy and the patient's overall health status should also be taken into account when considering different treatment opinions.

\section{Compliance with ethical standards}

Conflict of interest The authors declare that they have no conflict of interest.

\section{References}

1. Lai JS. Epidemiology of acute primary angle-closure glaucoma in the Hong Kong Chinese population: prospective study. Hong Kong Med J. 2001;7:118-23.

2. Seah SK, Foster PJ, Chew PT, Jap A, Oen F, Fam HB, et al. Incidence of acute primary angle-closure glaucoma in Singapore. An island-wide survey. Arch Ophthalmol. 1997;115: $1436-40$. 
3. Ramesh S, Maw C, Sutton CJ, Gandhewar JR, Kelly SP. Ethnic aspects of acute primary angle closure in a UK mulicultural conurbation. Eye. 2005;19:1271-5.

4. Ivanisevic M, Erceg M, Smoljanovic A, Trosic Z. The incidence and seasonal variations of acute primary angle-closure glaucoma. Coll Antropol. 2002;26:41-5.

5. Teikari J, Raivio I, Nurminen M. Incidence of acute glaucoma in Finland from 1973 to 1982. Graefes Arch Clin Exp Ophthalmol. 1987;225:357-60.

6. Baig N, Kam KW, Tham CC. Managing primary angle closure glaucoma - the role of lens extraction in this era. Open Ophthalmol J. 2016;10:86-93.

7. Ritch R, Liebmann JM. Argon laser peripheral iridoplasty. Ophthalmic Surg Lasers. 1996;27:289-300.

8. Ritch R. Argon laser treatment for medically unresponsive attacks of angle-closure glaucoma. Am J Ophthalmol. 1982;94:197-204.

9. Lam DS, Lai JS, Tham CC. Immediate argon laser peripheral iridoplasty as treatment for acute attack of primary angle-closure glaucoma: a preliminary study. Ophthalmology. 1998; 105:2231-6.

10. Tham CC, Lai JS, Lam DS. Immediate argon laser peripheral iridoplasty for acute attack of PACG (addendum to previous report). Ophthalmology. 1999;106:1042-3.

11. Lai JS, Tham CC, Lam DS. Limited argon laser peripheral iridoplasty as immediate treatment for an acute attack of primary angle closure glaucoma: a preliminary study. Eye. 1999; 13(Pt 1):26-30.

12. Lai JS, Tham CC, Chua JK, Lam DS. Immediate diode laser peripheral iridoplasty as treatment of acute attack of primary angle closure glaucoma: a preliminary study. J Glaucoma. 2001; 10:89-94.

13. Lam DS, Lai JS, Tham CC, Chua JK, Poon AS. Argon laser peripheral iridoplasty versus conventional systemic medical therapy in treatment of acute primary angle-closure glaucoma: a prospective, randomized, controlled trial. Ophthalmology. 2002; 109:1591-6.

14. Lai JS, Tham CC, Chua JK, Poon AS, Chan JC, Lam SW, et al. To compare argon laser peripheral iridoplasty (ALPI) against systemic medications in treatment of acute primary angle-closure: mid-term results. Eye. 2006;20:309-14.

15. Lam DS, Chua JK, Tham CC, Lai JS. Efficacy and safety of immediate anterior chamber paracentesis in the treatment of acute primary angle-closure glaucoma: a pilot study. Ophthalmology. 2002;109:64-70.

16. Lam DS, Tham CC, Lai JS, Leung DY. Current approaches to the management of acute primary angle closure. Curr Opin Ophthalmol. 2007;18:146-51.

17. Palmberg P. Slit lamp procedures in postoperative glaucoma management. In: Eye on the Bayou, New Concepts in Glaucoma, Cataract and Neuro-Opthalmology. In: Nussdorf JD, editors. The Hague, The Netherlands: Kugler Publications; 2006.

18. Liu W, Chen Y, Lv Y, Wang L, Xing X, Liu A, et al. Diode laser transscleral cyclophotocoagulation followed by phacotrabeculectomy on medically unresponsive acute primary angle closure eyes: the long-term result. BMC Ophthalmol. 2014;14:26.

19. Yusuf IH, Shah M, Shaikh A, James CB. Transscleral cyclophotocoagulation in refractory acute and chronic angle closure glaucoma. BMJ Case Rep. 2015;2015:bcr2015209552.

20. Williams AL, Moster MR, Rahmatnejad K, Resende AF, Horan T, Reynolds $\mathrm{M}$, et al. Clinical efficacy and safety profile of micropulse transscleral cyclophotocoagulation in refractory glaucoma. J Glaucoma. 2018;27:445-9.

21. Tan AM, Chockalingam M, Aquino MC, Lim ZI, See JL, Chew PT. Micropulse transscleral diode laser cyclophotocoagulation in the treatment of refractory glaucoma. Clin Exp Ophthalmol. 2010;38:266-72.
22. Luo L, Li M, Zhong Y, Cheng B, Liu X. Evaluation of secondary glaucoma associated with subluxated lens misdiagnosed as acute primary angle-closure glaucoma. J Glaucoma. 2013;22:307-10.

23. Lee YJ, Kang SM, Kang IB. Acute angle-closure glaucoma from spontaneous massive hemorrhagic retinal detachment. Korean J Ophthalmol. 2007;21:61-4.

24. Arthur SN, Mason J, Roberts B, Girkin CA. Secondary acute angle-closure glaucoma associated with vitreous hemorrhage after ruptured retinal arterial macroaneurysm. Am J Ophthalmol. 2004; 138:682-3

25. Liu YC, Lau LI, Lee FL, Ko YC, Hsu WM. Recurrent acute angle-closure attacks in age-related macular degenerationassociated massive posterior segment hemorrhage. Jpn J Ophthalmol. 2009;53:190-1.

26. Williams GS, Anderson L, Eddyshaw D. Macular hemorrhage as a cause of acute angle closure. Indian J Ophthalmol. 2013;61: 683-4.

27. Pesin SR, Katz LJ, Augsburger JJ, Chien AM, Eagle RC Jr. Acute angle-closure glaucoma from spontaneous massive hemorrhagic retinal or choroidal detachment. Ophthalmology. 1990;97:76-84.

28. Ming Zhi Z, Lim AS, Yin Wong T. A pilot study of lens extraction in the management of acute primary angle-closure glaucoma. Am J Ophthalmol. 2003;135:534-6.

29. Jacobi PC, Dietlein TS, Luke C, Engels B, Krieglstein GK. Primary phacoemulsification and intraocular lens implantation for acute angle-closure glaucoma. Ophthalmology. 2002;109: 1597-603.

30. Chen PP, Lin SC, Junk AK, Radhakrishnan S, Singh K, Chen TC. The effect of phacoemulsification on intraocular pressure in glaucoma patients: a report by the american academy of ophthalmology. Ophthalmology. 2015;122:1294-307.

31. Lam DS, Leung DY, Tham CC, Li FC, Kwong YY, Chiu TY, et al. Randomized trial of early phacoemulsification versus peripheral iridotomy to prevent intraocular pressure rise after acute primary angle closure. Ophthalmology. 2008;115:1134-40.

32. Husain R, Gazzard G, Aung T, Chen Y, Padmanabhan V, Oen FT, et al. Initial management of acute primary angle closure: a randomized trial comparing phacoemulsification with laser peripheral iridotomy. Ophthalmology. 2012;119:2274-81.

33. Radhakrishnan S, Chen PP, Junk AK, Nouri-Mahdavi K, Chen TC. Laser peripheral iridotomy in primary angle closure: a report by the American Academy of Ophthalmology. Ophthalmology. 2018;125:1110-20.

34. Imaizumi M, Takaki Y, Yamashita H. Phacoemulsification and intraocular lens implantation for acute angle closure not treated or previously treated by laser iridotomy. J Cataract Refract Surg. 2006;32:85-90.

35. Moghimi S, Hashemian H, Chen R, Johari M, Mohammadi M, Lin SC. Early phacoemulsification in patients with acute primary angle closure. J Curr Ophthalmol. 2015;27:70-5.

36. Fleck BW, Wright E, Fairley EA. A randomised prospective comparison of operative peripheral iridectomy and Nd:YAG laser iridotomy treatment of acute angle closure glaucoma: 3 year visual acuity and intraocular pressure control outcome. $\mathrm{Br} \mathrm{J}$ Ophthalmol. 1997;81:884-8.

37. Saunders DC. Acute closed-angle glaucoma and Nd-YAG laser iridotomy. Br J Ophthalmol. 1990;74:523-5.

38. Buckley SA, Reeves B, Burdon M, Moorman C, Wheatcroft S, Edelsten $\mathrm{C}$, et al. Acute angle closure glaucoma: relative failure of YAG iridotomy in affected eyes and factors influencing outcome. Br J Ophthalmol. 1994;78:529-33.

39. Playfair TJ, Watson PG. Management of acute primary angleclosure glaucoma: a long-term follow-up of the results of peripheral iridectomy used as an initial procedure. $\mathrm{Br} \mathrm{J}$ Ophthalmol. 1979;63:17-22. 
40. Krupin T, Mitchell KB, Johnson MF, Becker B. The long-term effects of iridectomy for primary acute angle-closure glaucoma. Am J Ophthalmol. 1978;86:506-9.

41. Boey PY, Singhal S, Perera SA, Aung T. Conventional and emerging treatments in the management of acute primary angle closure. Clin Ophthalmol. 2012;6:417-24.

42. Tomey KF, Traverso CE, Shammas IV. Neodymium-YAG laser iridotomy in the treatment and prevention of angle closure glaucoma. A review of 373 eyes. Arch Ophthalmol. 1987;105: 476-81.

43. Ang LP, Ang LP. Current understanding of the treatment and outcome of acute primary angle-closure glaucoma: an Asian perspective. Ann Acad Med Singapore. 2008;37:210-5.

44. Ho T, Fan R. Sequential argon-YAG laser iridotomies in dark irides. Br J Ophthalmol. 1992;76:329-31.

45. Aung T, Ang LP, Chan SP, Chew PT. Acute primary angle-closure: long-term intraocular pressure outcome in Asian eyes. Am J Ophthalmol. 2001;131:7-12.

46. Aung T, Friedman DS, Chew PT, Ang LP, Gazzard G, Lai YF, et al. Long-term outcomes in asians after acute primary angle closure. Ophthalmology. 2004;111:1464-9.

47. Lim LS, Aung T, Husain R, Wu YJ, Gazzard G, Seah SK. Acute primary angle closure: configuration of the drainage angle in the first year after laser peripheral iridotomy. Ophthalmology. 2004;111:1470-4.

48. Choong YF, Irfan S, Menage MJ. Acute angle closure glaucoma: an evaluation of a protocol for acute treatment. Eye. 1999;13(Pt 5):613-6.

49. Tan AM, Loon SC, Chew PT. Outcomes following acute primary angle closure in an Asian population. Clin Exp Ophthalmol. 2009;37:467-72.

50. Lai JS, Tham CC, Chan JC. The clinical outcomes of cataract extraction by phacoemulsification in eyes with primary angleclosure glaucoma (PACG) and co-existing cataract: a prospective case series. J Glaucoma. 2006;15:47-52.

51. Shingleton BJ, Chang MA, Bellows AR, Thomas JV. Surgical goniosynechialysis for angle-closure glaucoma. Ophthalmology. 1990;97:551-6.

52. Teekhasaenee C, Ritch R. Combined phacoemulsification and goniosynechialysis for uncontrolled chronic angle-closure glaucoma after acute angle-closure glaucoma. Ophthalmology. 1999;106:669-74. discussion674-665.

53. Harasymowycz PJ, Papamatheakis DG, Ahmed I, Assalian A, Lesk M, Al-Zafiri Y, et al. Phacoemulsification and goniosynechialysis in the management of unresponsive primary angle closure. J Glaucoma. 2005;14:186-9.
54. Lai JS, Tham CC, Lam DS. The efficacy and safety of combined phacoemulsification, intraocular lens implantation, and limited goniosynechialysis, followed by diode laser peripheral iridoplasty, in the treatment of cataract and chronic angle-closure glaucoma. J Glaucoma. 2001;10:309-15.

55. Lai JS, Tham CC, Chua JK, Lam DS. Efficacy and safety of inferior 180 degrees goniosynechialysis followed by diode laser peripheral iridoplasty in the treatment of chronic angle-closure glaucoma. J Glaucoma. 2000;9:388-91.

56. Sihota R, Goyal A, Kaur J, Gupta V, Nag TC. Scanning electron microscopy of the trabecular meshwork: understanding the pathogenesis of primary angle closure glaucoma. Indian $\mathbf{J}$ Ophthalmol. 2012;60:183-8.

57. Aung T, Tow SL, Yap EY, Chan SP, Seah SK. Trabeculectomy for acute primary angle closure. Ophthalmology. 2000;107:1298302.

58. Tham CC, Kwong YY, Baig N, Leung DY, Li FC, Lam DS. Phacoemulsification versus trabeculectomy in medically uncontrolled chronic angle-closure glaucoma without cataract. Ophthalmology. 2013;120:62-7.

59. Tham CC, Kwong YY, Leung DY, Lam SW, Li FC, Chiu TY, et al. Phacoemulsification versus combined phacotrabeculectomy in medically uncontrolled chronic angle closure glaucoma with cataracts. Ophthalmology. 2009;116:725-31. 731 e721-3

60. Hollander DA, Pennesi ME, Alvarado JA. Management of plateau iris syndrome with cataract extraction and endoscopic cyclophotocoagulation. Exp Eye Res. 2017;158:190-4.

61. Wang JC, Chew PT. What is the direct cost of treatment of acute primary angle closure glaucoma? The Singapore model. Clin Exp Ophthalmol. 2004;32:578-83.

62. Chan PP, Li EY, Tsoi KKF, Kwong YY, Tham CC. Cost-effectiveness of phacoemulsification versus combined phacotrabeculectomy for treating primary angle closure glaucoma. J Glaucoma. 2017;26:911-22.

63. Azuara-Blanco A, Burr J, Ramsay C, Cooper D, Foster PJ, Friedman DS, et al. Effectiveness of early lens extraction for the treatment of primary angle-closure glaucoma (EAGLE): a randomised controlled trial. Lancet. 2016;388:1389-97.

64. Wittenborn JS, Rein DB. Cost-effectiveness of glaucoma interventions in Barbados and Ghana. Optom Vis Sci. 2011;88:155-63.

65. Kymes SM, Plotzke MR, Kass MA, Boland MV, Gordon MO. Effect of patient's life expectancy on the cost-effectiveness of treatment for ocular hypertension. Arch Ophthalmol. 2010;128: 613-8.

66. Chan PP, Li EY, Tham CC. Cost-effectiveness in the Treatment of Glaucoma. US Ophthalmic Rev. 2014;7:131-6. 